\title{
THE FIBRE OF A CELL ATTACHMENT
}

\author{
by STEPHEN HALPERIN and JEAN-MICHEL LEMAIRE
}

(Received 5th October 1993)

\begin{abstract}
In view of understanding the Hopf algebra structure of the loop space homology $H_{*}\left(\Omega\left(E \bigcup_{f} e^{n+1}\right)\right)$ in terms of $H_{*}(\Omega E)$ and the map $f$, we consider the homotopy fibre $F$ of the inclusion map $\omega: E \hookrightarrow E \bigcup_{f} e^{n+1}$. In [15], the case when $H_{*}(\Omega \omega)$ is surjective (the "inert" case) was studied, and in [11] a weaker condition, called "lazy", was considered. Here we give several new characterizations of inert and lazy cell attachments in terms of properties of $F$. We also show how these results extend to the case of the mapping cone $E U_{S} C W$ of an arbitrary map $f: W \rightarrow E$.
\end{abstract}

1991 Mathematics Subject Classification. Primary 55P35, 55R20, 55P62; Secondary 16W30, 18G15.

\section{Introduction}

In this paper we work over a fixed ground field $\kappa$ of characteristic $p \geqq 0$; in particular we write $H_{*}(-)=H_{*}(-; \kappa)$. We restrict consideration to spaces $X$ such that $H_{k}(X ; \mathbb{Z}(p))$ is a finitely generated $\mathbb{Z}(p)$-module for each $k$.

Now suppose $f: S^{n} \rightarrow E$ is a continuous map from the n-sphere to a 1-connected $c w$-complex $E$, with $n \geqq 2$. The space $E \bigcup_{f} e^{n+1}$ is said to be obtained by attaching a $(n+1)$-cell to $E$ along $f$. In this paper we pursue the quest for a better understanding of the Hopf algebra structure of the loop space homology $H_{*}\left(\Omega\left(E \bigcup_{f} e^{n+1}\right)\right)$ in terms of $H_{*}(\Omega E)$ and the map $f$; earlier results can be found viz. in $[11,15,14]$.

Our approach here is to consider the homotopy fibre $F$ of the inclusion map $E \hookrightarrow E \bigcup_{S} e^{n+1}$. Indeed for any fibration sequence $F \rightarrow E \rightarrow B$, there is a homotopy action $v: \Omega B \times F \rightarrow F$, often called the holonomy of the fibration. A remarkable feature of the holonomy action when $B=E \bigcup_{f} e^{n+1}$ is that the reduced homology of the fibre $H_{+}(F)$ is a free module over $H_{*}\left(\Omega\left(E \bigcup_{f} e^{n+1}\right)\right)$ : this was observed and proved by Félix and Thomas [12].

Our first result, proven in Section 1, is that this property characterizes fibrations of the form $F \rightarrow E \rightarrow E \bigcup_{f} e^{n+1}$ :

Proposition 1.1. Let $F \nrightarrow E \leftrightarrow B$ be a fibration sequence of 1-connected cw-complexes. The following statements are equivalent:

(1) As a $H_{*}(\Omega B)$-module, $H_{+}(F)$ is freely generated by a single element in $H_{n}(F), n \geqq 2$.

Research of the first-named author was partially supported by an NSERC operating grant.

The second-named author was fully supported by the CNRS.

This research was partially supported by a NATO travel grant. 
(2) When the fibration sequence is localized at $p$, there exists $a \in \pi_{n}(F)$ such that the obvious extension $\theta: E \bigcup_{\lambda \alpha} e^{n+1} \rightarrow B$ of $\omega$ is a p-equivalence.

Now consider a fixed attachment $E \rightarrow E \bigcup_{f} e^{n+1}$, write $B=E \bigcup_{f} e^{n+1}$, and let

$$
F \rightarrow E \rightarrow B
$$

be the corresponding fibration sequence. In [15] the case when $H_{*}(\Omega E) \rightarrow H_{*}(\Omega B)$ is surjective was extensively studied: in that case the attaching map $f$ is said to be p-inert. Let $\delta: \Omega B \rightarrow F$ be the connecting map in the Eckmann-Hilton sequence, i.e. the holonomy evaluated on the base point. A condition weaker than inertia was introduced in [11]: $f$ is said to be p-lazy (or lazy if $p$ is clear from the context) if $H_{*}(\delta): H_{*}(\Omega B) \rightarrow H_{*}(F)$ is trivial.

There do exist lazy attachments which are not inert, but the only examples we know are "unreasonable" in the sense that the attachment also kills the attaching map of a cell of higher dimension in $E$. This situation will be discussed in a forthcoming paper. Here we shall establish new characterizations of $p$-inert and $p$-lazy attaching maps in terms of the fibre $F$.

Let us first recall ([15, Prop. 3.4]) that in the rational case $(p=0)$, the attaching map $f$ is inert iff the fibre $F$ has the rational homotopy type of a wedge of spheres or, equivalently, if the rational homotopy Lie algebra of $F$ is free. In Section 3 we prove the following generalization of this result to the case $p>0$ :

Proposition 1.2. (1) The attaching map $f$ is p-inert if and only if the Pontryagin algebra $H_{*}(\Omega F)$ is a tensor algebra.

(2) The attaching map $f$ is p-lazy if and only if all cup-products in $H^{*}(F)$ vanish.

Remark 1.3. The characteristic property (1) has already been established for $p>2$ by Félix and Thomas [14].

Remark 1.4. A space whose loop space homology is a free algebra (in particular a wedge of spheres) has no non-trivial cup-products. This is a classical and easy consequence [3] of the theory of Adams-Hilton models, which we shall review below. Thus Proposition 1.2 provides another way of seeing that inertia implies laziness.

The proof of Proposition 1.2(2) uses the following fact, which we mention here as having some independent interest.

Proposition 1.5. Let $\hat{f} \in \pi_{n}(\Omega E)$ be the adjoint of the attaching map $f$. Then its Hurewicz image in $H_{n}(\Omega E)$ vanishes if and only if

$$
H_{*}(\delta): H_{*}(\Omega B) \rightarrow H_{*}(F)
$$

is surjective. 
When the attaching map $f$ is inert, the algebra $H_{*}(\Omega F)$, which is free by $1.2(1)$, is the Hopf kernel of the map $H_{*}(\Omega \omega): H_{*}(\Omega E) \rightarrow H_{*}(\Omega B)$. In Section 4 , we give an example of a non-inert map such that $H_{*}(\Omega \omega)$ still has a free Hopf kernel. We also show examples of non-homotopic inert attachments with homotopic holonomy actions.

Rationally, the map $\Omega \omega: \Omega E \rightarrow \Omega B$ admits a section iff it induces a surjection in homology or in homotopy, because rational loop spaces split as products of $K(\mathbb{Q}, n)$ 's. Thus over the rationals the attachment is inert iff $\delta$ is null homotopic. We extend this observation to a field of any characteristic in terms of Adams-Hilton models: recall [1, 3] that an Adams-Hilton model for a simply-connected space $X$ is a quasi-isomorphism from a differential graded tensor algebra $A H(X)=(T(V), d)$ to the chain algebra $C_{*}(\Omega X)$. A continuous map $\phi: X \rightarrow Y$ determines a unique DGA homotopy class of chain algebra morphisms $A H(\phi): A H(X) \rightarrow A H(Y)$, representing $C_{*}(\Omega \phi)$. In particular, $H(A H(\phi))=$ $H_{*}(\Omega \phi)$. Now it follows from [2] and [4] that over the rationals $\phi \sim \psi$ if and only if $A H(\phi) \sim A H(\psi)$. Thus over the rationals the attachment is inert if and only if $A H(\delta)$ is null-homotopic; we generalise this to any characteristic:

Proposition 1.6. Assume $B$ is 2-connected. The attaching map $f$ is p-inert if and only if $A H(\delta)$ is homotopically trivial.

This proposition is proven in Section 5 , and it provides another way of seeing that inertia implies laziness. Indeed if $A H(\delta)$ is homotopically trivial, then $H_{+}(\delta)=0$, i.e. $f$ is p-lazy.

Next, recall that the depth of a connected $\kappa$-algebra $A$ is either the least integer $d$ such that $\operatorname{Ext}_{A}^{d}(\kappa, A) \neq 0$, or else $\infty$ if no such integer exists. By extension, if $X$ is a 1-connected space, we set depth $(X ; p)=\operatorname{depth} H_{*}\left(\Omega X, \mathbb{F}_{p}\right)$.

In Section 6, we prove the following result, which is probably not the best possible:

Proposition 1.7. Assume that $B$ is a finite complex whose cohomology algebra $H^{*}\left(B: \mathbb{F}_{p}\right)$ is not generated-as an algebra-by a single element. If $\operatorname{depth}(F ; p)$ is finite, then $f$ is p-lazy.

In a final section, we observe that the notions of inertia and laziness can readily be extended to maps $f: W \rightarrow E$ between arbitrary 1-connected spaces: let $B=C_{f}=E \bigcup_{f} C W$ be the mapping cone of $f$, let $F$ be the homotopy fibre of the inclusion $g: E \hookrightarrow C_{j}$, and let $\delta: \Omega B \rightarrow F$ be the connecting map.

Definition 1.8. We say that $f$ is p-inert (resp. p-lazy) if $H_{*}(A g): H_{*}(\Omega E) \rightarrow H_{*}(\Omega B)$ is surjective (resp. $0=H_{+}(\delta): H_{+}(\Omega B) \rightarrow H_{+}(F)$ ).

In this general setting, $H_{+}(F)$ is still a free $H_{*}(\Omega B)$-module for any $W$ and $f$, as follows from a more recent result of Félix and Thomas [13]. We show that Proposition 1.7 also holds in this context, and that Propositions 1.2 and 1.6 also hold provided $W$ is a suspension, or more generally an "AH-suspension", i.e. $H_{*}(\Omega W)$ is a tensor algebra. 
The second-named author wishes to thank the University of Toronto for hospitality during the early stage of the preparation of this paper.

\section{Proof of Proposition 1.1}

We recall from the introduction that the implication $(2) \Rightarrow(1)$ is due to Félix and Thomas [12]. Here we give a simple proof of the converse, namely Proposition 2.1 below; however, we note that, when we communicated this result to Félix and Thomas, they realized and pointed out to us that it also follows easily from [13, Theorem 1].

Proposition 2.1. Let $F \stackrel{\rightarrow}{\rightarrow} \leftrightarrow B$ be a fibration sequence of 1-connected p-local cw-complexes, and assume that $H_{+}(F)$ is a free $H_{*}(\Omega B)$-module on a single generator in $H_{n}(F), n \geqq 1$. Then there exists $a \in \pi_{n}(F)$ whose Hurewicz image $h(a)$ is such a generator, and the extension $\theta: B^{\prime}=E \bigcup_{\lambda \alpha} e^{n+1} \rightarrow B$ of $\omega$ is a homotopy equivalence.

The following definition and lemma embody the main idea of the proof. The proof of the lemma is straightforward and left to the reader.

Definition 2.2. Let $g: V_{*} \rightarrow W_{*}$ be a morphism of graded vector spaces. We say that $g$ is an almost isomorphism of degree $n$ if either $g$ is surjective with Ker $g$ 1-dimensional and concentrated in degree $n-1$, or else $g$ is injective with Coker $g$ 1-dimensional and concentrated in degree $n$.

Lemma 2.3. If $g: V_{*} \rightarrow W_{*}$ is an almost isomorphism at degree $n$ of differential graded vector spaces, then $H(g)$ is an almost isomorphism at degree $n$.

We now recall that any fibration sequence $F \rightarrow E \rightarrow B$ of 1-connected spaces gives rise to a first quadrant Eilenberg-Moore spectral sequence ([19]) such that

$$
E_{p, q}^{2}=\operatorname{Tor}_{p, q}^{H_{*}(\Omega B)}\left(\kappa, H_{*}(F)\right) \Rightarrow H_{p+q}(E)
$$

where the $H_{*}(\Omega B)$-module structure of $H_{*}(F)$ is given by the holonomy.

Consider the following morphism of fibration sequences:

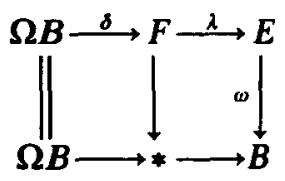

It induces a morphism of spectral sequences

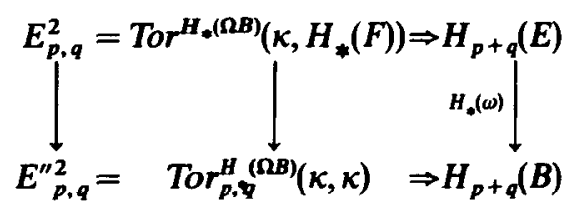


Lemma 2.4. The map $E_{p, q}^{2} \rightarrow E_{p, q}^{\prime 2}$ is an almost isomorphism at degree $n+1$.

Proof. In the extension of $H_{*}(\Omega B)$-modules:

$$
0 \rightarrow H_{+}(F) \rightarrow H_{*}(F) \rightarrow \kappa \rightarrow 0
$$

the kernel is a free module on a single generator $h(a)$ of degree $n$; this extension determines a long exact sequence for $\operatorname{Tor}_{p, q}^{H_{,}(\Omega B)}(\kappa,-)$ which yields $\operatorname{Tor}_{p, q}^{H_{,}(\Omega B)}\left(\kappa, H_{*}(F)\right)=$ $\operatorname{Tor}_{p, q}^{H,(\Omega B)}(\kappa, \kappa)$ for $p \geqq 2$ and whose bottom part reads

$$
\begin{aligned}
& 0 \rightarrow \operatorname{Tor}_{1, *}^{H_{*}(\Omega B)}\left(\kappa, H_{*}(F)\right) \rightarrow \operatorname{Tor}_{1 ; *}^{H,(\Omega B)}(\kappa, \kappa) \\
& \kappa \bigotimes_{\left.H_{*}(\Omega B)\right)}^{\bigotimes} H_{+}(F) \rightarrow \kappa \bigotimes_{H_{*}(\Omega B)}^{\bigotimes} H_{*}(F) \rightarrow \kappa \rightarrow 0
\end{aligned}
$$

But $\kappa \bigotimes_{H_{*}(\Omega B)} H_{+}(F)=\kappa \cdot h(a)$. Therefore, the map

$$
\operatorname{Tor}_{*, *}^{H,(\Omega B)}(\kappa, H *(F)) \rightarrow \operatorname{Tor}_{* * *}^{H,(\Omega B)}(\kappa, \kappa)
$$

is either surjective with one-dimensional kernel in bidegree $(0, n)$ or injective with one-dimensional cokernel in bidgree $(1, n)$.

Lemma 2.5. Let $\mathrm{g}: V \rightarrow W$ be a morphism of filtered vector spaces, and assume both filtrations are bounded below and cocomplete. Then if $E^{0} g$ is an almost isomorphism, so is g.

Proof of Proposition 2.1. Since $H_{+}(F) \cong H_{*}(\Omega B) \otimes \kappa . \alpha$ with $\alpha \in H_{n}(F)$, one has $\operatorname{dim} H_{n}(F)=1$ and $H_{i}(F)=0$ for $0<i<n$. Since $F$ is assumed to be $p$-local and of finite type, one has $H_{i}(F ; \mathbb{Z})=0$ for $0<i<n$ and $H_{n}(F)=H_{n}(F ; \mathbb{Z}) \otimes \kappa$. Therefore there exists $a \in \pi_{n}(F)$ such that $h(a) \otimes 1 \neq 0$ in $H_{n}(F)$. Now Lemmas 2.4 and 2.5 imply that $H_{*}(\omega)$ is an almost isomorphism at degree $n+1$. But clearly so is $H_{*}(E) \rightarrow H_{*}\left(E \bigcup_{\lambda a} e^{n+1}\right)$. In particular both maps are isomorphisms for $*>n+1$ and $*<n$, and therefore so is $H_{*}(\theta)$. Thus we are left to show that $H_{n}(\theta)$ and $H_{n+1}(\theta)$ are isomorphisms.

Recall that $B^{\prime}=E \bigcup_{\lambda a} e^{n+1}$, and let $F^{\prime}$ be the homotopy fibre of the inclusion $E \subset B^{\prime}$. Consider the morphism of fibre sequences

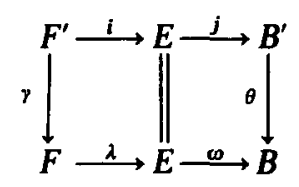

which induces a morphism of Serre exact sequences 


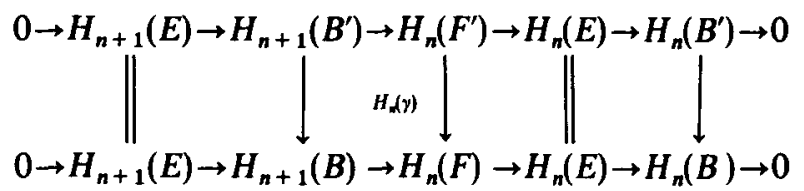

By standard diagram chasing, it suffices to prove that the middle vertical map $H_{n}(\gamma)$ is an isomorphism, and since it is a map between vector spaces of dimension 1 , that it is surjective. But $a: S^{n} \rightarrow F$ factors through $F^{\prime}$, and so $h(a) \otimes 1$ is in the image of $H_{n}(\gamma)$.

\section{Inert and lazy attachments and their fibres}

In this section we prove Proposition 1.2. For the convenience of the reader, we begin by recalling some basic facts about Adams-Hilton models and Hopf algebras, which we shall use in the proof and later.

(3.1). Adams-Hilton models. An Adams-Hilton model $A H(X)$ of a 1-connected space $X$ is a differential graded tensor algebra $(T(V), d)$ quasi-isomorphic to the chain algebra $C_{*}(\Omega X)$. Let $T^{k}(V)$ be the $k$ th tensor power, and let

$$
d_{1}: V \stackrel{d}{\longrightarrow} T \geqq 1(V) \stackrel{p}{\longrightarrow} T \geqq 1(V) / T^{\geqq 2}(V)=V
$$

be the "linear part" of the differential $d$. Then $H\left(V, d_{1}\right)$ is naturally isomorphic to the desuspension of the reduced homology $H_{+}(X)$. If $d_{1}=0, A H(X)$ is said to be minimal: one can always replace a non-minimal model by a minimal one, which is then unique up to isomorphism. Moreover the "quadratic part" $d_{2}: V \rightarrow V \otimes V$ of the differential $d$ in a minimal $A H(X)$ coincides up to suspensions with the reduced diagonal $H_{+}(X) \rightarrow$ $H_{+}(X) \otimes H_{+}(X)$. Finally, when $f \in \pi_{n}(X)$ and $Y=X \bigcup_{f} e^{n+1}$, one can choose $A H(Y)=$ $T(V \oplus \kappa \cdot w), D)$, where $D_{\mid V}=d_{\mid V}$ and $D w$ is a cycle in $T(V)$ which represents the Hurewicz image of the adjoint of $f$.

The following lemma easily follows from the properties of the Adams-Hilton model and of the bar and cobar constructions:

Lemma 3.2. Let $X$ be a 1-connected cw-complex of finite type. The following conditions are then equivalent:

(i) The Pontryagin algebra $H_{*}(\Omega X)$ is a tensor algebra.

(ii) The differential in the minimal Adams-Hilton model $A H(X)$ is zero.

(iii) The multiplication in $H^{+}(X)$ is trivial and the $D G A, C^{*}(X)$, is quasi-isomorphic to $H^{*}(X)$, endowed with the zero differential.

When these conditions are satisfied, one has $H_{*}(\Omega X) \cong T\left(s^{-1} H_{+}(X)\right)$.

Definition 3.3. A space which satisfies the conditions of Lemma 3.2. is called an AH-suspension (at p). 
Note that any suspension is an AH-suspension at every prime by the Bott-Samelson theorem, and so is a co-H-space $Z$ since it is a retract of $\Sigma \Omega Z$. A simply connected space whose homology (with coefficients in $\kappa$ ) is concentrated in odd degrees is an AHsuspension by (ii), since its Adams-Hilton model is concentrated in even degrees.

(3.4). Hopf algebras. In this paper, a Hopf algebra will always mean a cocommutative connected graded Hopf $\kappa$-algebra. We refer to $[18,20]$ for more information about such Hopf algebras.

A Hopf algebra can be viewed as a group object in the category of cocommutative connected $\kappa$-coalgebras, and standard group theoretic notions can be given sense for Hopf algebras; in particular, each Hopf algebra $A$ admits an inverse map, namely the unique coalgebra map $\sigma: A \rightarrow A$ such that

$$
m \circ(A \otimes \sigma) \circ \Delta=\Delta \circ(\sigma \otimes A) \circ m=\varepsilon
$$

where $m: A \otimes A \rightarrow A$ is the multiplication, $\Delta: A \rightarrow A \otimes A$ is the diagonal and $\varepsilon$ is the augmentation. A map of coalgebras is defined by the composition

$$
A \otimes A \stackrel{\Delta \otimes A}{\longrightarrow} A \otimes A \otimes A \stackrel{A \otimes T}{\longrightarrow} A \otimes A \otimes A \stackrel{A \otimes A \otimes a}{\longrightarrow} A \otimes A \otimes A \stackrel{m_{3}}{\longrightarrow} A
$$

where $T$ is the interchange map and $m_{3}$ multiplication of the three factors. It is denoted $a \otimes b \mapsto \operatorname{Ad} a(b)$. Note that if $a \in A$ is primitive, $\sigma a=-a$ and $\operatorname{Ad} a=[a,-]$. The map $a \mapsto \operatorname{Ad} a$ is a representation of $A$ into itself, called the adjoint representation.

A sub-Hopf algebra $B \subset A$ is normal (resp. central) if the operators Ad $a, a \in A_{+}$ preserve $B$ (resp. are zero on $B$ ).

Given a normal sub-Hopf algebra $B \subset A$ there is a unique Hopf algebra structure on $A \bigotimes_{B} \kappa$ such that the surjection is a Hopf algebra morphism; this quotient Hopf algebra is denoted by $A / / B$. Given any Hopf morphism $\phi: A \rightarrow A^{\prime}$ there is a unique normal subHopf algebra $B \subset A$ such that $\operatorname{Im} \phi=A / / B ; B$ is called the Hopf kernel of $\phi$.

With any Hopf algebra $A$ is associated its derived series

$$
A \supset A^{[1]} \supset \cdots A^{[s]} \supset \cdots
$$

of normal sub-Hopf algebras. A Hopf algebra $A$ is solvable if $A^{[t]}=\kappa$ for some $t$. The radical $R$ of $A$ is the least normal sub-Hopf algebra which contains all normal solvable sub-Hopf algebras of $A$.

The following proposition is an enhanced version of Part (1) of 1.2 (compare [14, Theorems 1 and 2]):

Proposition 3.5. Let $F$ be the fibre of the inclusion $E \hookrightarrow E \bigcup_{f} e^{n+1}$. The following statements are equivalent:

(i) The algebra $H_{*}(\Omega F)$ is a tensor algebra, i.e. $F$ is an $A H$-suspension.

(ii) The Hopf algebra $H_{*}(\Omega F)$ either has no radical, or is isomorphic to a tensor algebra on a single generator. 
(iii) The map $H_{*}(\Omega \omega)$ is surjective, i.e. $f$ is p-inert.

Proof. (i) $\Rightarrow$ (ii) because any sub-Hopf algebra of a free Hopf algebra is free.

(ii) $\Rightarrow$ (iii): let $\lambda: F \rightarrow E$ be the inclusion of the fibre; we recall ([10]) that the Hopf kernel $K$ of $H_{*}(\Omega \lambda)$ is central in $H_{*}(\Omega F)$. If the latter is a free algebra on more than one generator, its centre is trivial; hence $K=\kappa$ and $H_{*}(\Omega \lambda)$ is injective. Now by a classical argument, the Serre spectral sequence of the fibration $\Omega F \stackrel{\lambda}{\rightarrow} \Omega E \stackrel{\leftrightarrow}{\rightarrow} \Omega B$ collapses at the $E^{2}$ level and that map $H_{*}(\Omega \omega)$ is surjective, i.e. (iii) holds. If $H_{*}(\Omega F) \cong T(a)$, then by 3.2 one has $\operatorname{dim} H_{+}(F)=1$, since $H_{+}(F)$ is a free $H_{*}(\Omega B)$-module, one must have $H_{*}(\Omega B)=\kappa$ and (iii) trivially holds.

(iii) $\Rightarrow$ (i) If $H_{*}(\Omega \omega)$ is surjective, the Serre spectral sequence of the multiplicative fibration $\Omega F \rightarrow \Omega F \rightarrow \Omega B$ again collapses at the $E^{2}$ level and thus $H_{*}(\Omega F)$ is the Hopf kernel of $H_{*}(\Omega \omega)$. By [15] (actually only the characteristic zero case is discussed in this paper, but the arguments readily apply to cocommutative Hopf algebras over any field), this kernel is a free Hopf algebra whose module of indecomposables is a free $H_{*}(\Omega B)$ module. In particular (ii) holds.

Thus part (1) of Proposition 1.2 is proven.

\section{(3.6). Proof of Proposition 1.2.}

Let $X$ be a 1-connected space. Recall that $H_{\star}(X)$ is a commutative coalgebra with diagonal $\Delta$. Given $\alpha \in H_{*}(X ; \kappa)$, we shall write

$$
\Delta \alpha=\alpha \otimes 1+1 \otimes \alpha+\sum \alpha^{\prime} \otimes \alpha^{\prime \prime}
$$

with $\alpha^{\prime}, \alpha^{\prime \prime} \in H_{+}(X ; \kappa)$.

Let us assume $f$ is $p$-lazy, namely that $H_{*}(\Omega B) \rightarrow H_{*}(F)$ is trivial. Since $H_{+}(F)$ is a free $H_{*}(\Omega B)$-module, every element in $H_{+}(F)$ can be uniquely written $\alpha . a$ with $\alpha \in H_{*}(\Omega B)$ and $a$ being the generator in $H_{n}(F)$. On the other hand, $\alpha \cdot 1=0$ for every $\alpha \in H_{+}(\Omega B)$. Since the holonomy $H_{*}(\Omega B) \otimes H_{*}(F) \rightarrow H_{*}(F)$ is a map of coalgebras, we have

$$
\begin{aligned}
\Delta(\alpha \cdot a) & =\left(\alpha \otimes 1+1 \otimes \alpha+\sum \alpha^{\prime} \otimes \alpha^{\prime \prime}\right)(a \otimes 1+1 \otimes a) \\
& =\alpha \cdot a \otimes 1+1 \otimes \alpha \cdot a .
\end{aligned}
$$

Therefore every element in $H_{+}(F)$ is primitive.

Conversely, let us assume that every element in $H_{+}(F)$ is primitive, and let us show that $\alpha \cdot 1=0$ for all $\alpha \in H_{+}(\Omega B)$. Otherwise, there would exist an element $\beta$ of least degree such that $\beta \cdot 1 \neq 0$. We get

$$
\begin{aligned}
\Delta(\beta \cdot a) & =\left(\beta \otimes 1+1 \otimes \beta+\sum \beta^{\prime} \otimes \beta^{\prime \prime}\right)(a \otimes 1+1 \otimes a) \\
& =\beta \cdot a \otimes 1+1 \otimes \beta \cdot a+\beta \cdot 1 \otimes a+(-1)^{n|\beta|} a \otimes \beta \cdot 1 .
\end{aligned}
$$


Since $\beta \cdot a$ is primitive, one must have $\beta \cdot 1 \otimes a+(-1)^{n|\beta|} a \otimes \beta \cdot 1=0$ : this can only occur if $\beta \cdot 1=\lambda a$ for some $\lambda \in \kappa$ and $(-1)^{n|\beta|}+1=0$. But since $H_{+}(F)$ is $H_{*}(\Omega B)$-free on the single generator $a$, the map $\delta_{*}: H_{+}(\Omega B) \rightarrow H_{*}(F)$, which is the evaluation on 1 , would be surjective.

By Proposition 1.5, which we shall prove below, this would imply that the element $\phi \in H_{*}(\Omega E)$ which corresponds to $f$ is zero. Then the Adams-Hilton model of $B$ would be isomorphic to $T(V) \bigsqcup T(b)$, where $T(V)$ is the Adams-Hilton model of $E$ and $d b=0$, $|b|=n$. Therefore one would have $H_{*}(\Omega B) \cong H_{*}(\Omega E) \bigsqcup T(b)$. Now let us observe that the Serre spectral sequence for the principal fibre sequence $\Omega E \rightarrow \Omega B \stackrel{\delta}{\rightarrow} F$ must collapse at the $E^{2}$ level, because $H_{*} \delta$ ) is surjective (or because $H_{*}(\omega)$ is injective!). Therefore we have an isomorphism of coalgebras $H_{*}(F) \cong \kappa \bigotimes_{H_{*}(\Omega E)} H_{*}(\Omega B)$. Using the coproduct structure of the algebra $H_{*}(\Omega B)$, one sees by inspection that the class in $H_{*}(F)$ of either $b^{2}$ or $b^{3}$ has a non trivial diagonal, which contradicts our hypothesis.

We are left to prove Proposition 1.5 , namely that $\delta_{*}: H_{*}(\Omega B) \rightarrow H_{*}(F)$ is surjective if and only if the element $\phi \in H_{*}(\Omega E)$ which corresponds to $f$ is zero.

Proof. We consider the following morphism of fibration sequences:

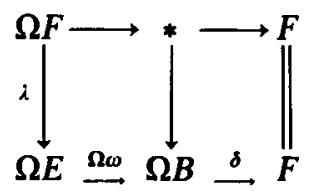

and the induced morphism of Serre spectral sequences; in the upper one $a \in H_{n}(F)$ transgresses to the generator of $H_{n-1}(\Omega F)$, while in the lower one it transgresses to $\phi$. Now if $H_{*}(\delta)$ is surjective, the lower spectral sequence collapses as we already observed; hence the transgression is zero and $\phi=0$. Conversely, if $\phi=0, a$ is a permanent cycle and therefore is in the image of $H_{*}(\delta)$; but this means that there exists $\alpha \in H_{*}(\Omega B)$ such that $\alpha \cdot 1=a$, and so $H_{*}(\delta)$ is surjective.

\section{Some examples}

By Proposition 1.2(1), the attaching map $f$ is inert if and only if the algebra $H_{*}(\Omega F)$ is free, and then the latter is the Hopf kernel of the map $H_{*}(\Omega \omega): H_{*}(\Omega E) \rightarrow H_{*}(\Omega B)$. One may ask whether the attachment is inert if the Hopf kernel of $H_{*}(\Omega \omega)$ is free. Here is a counter-example in characteristic zero. We shall use the notation

$$
\underset{\underline{\pi}}{\underline{n}}(X)=P H_{*}(\Omega X ; \mathbb{Q}), P(-)=\text { primitive subspace }
$$

for the rational homotopy Lie algebra of the space $X$, and we shall work up to rational equivalence. 
Example 4.1. Let $M$ be the coformal space with

$$
\underset{\underline{\pi}}{\underline{\pi}}(M)=\mathbb{L}(a, b) /([a,[a, b]],[b,[a, b]])=L, \operatorname{deg} a=\operatorname{deg} b=2
$$

Its Sullivan model is the cochain algebra on $\pi(M)$, i.e. is the exterior algebra on generators $x, y, z$ of degrees $3,3,5$ with $d z=x y$. One checks from this that $M$ fibres over $S^{3} \times S^{3}$ with fibre $S^{5}$. The homology decomposition of $M$ is therefore of the form $S^{3} \vee S^{3} \cup\left(e^{8} \vee e^{8}\right) \cup e^{11}$. Moreover the attaching maps of the 8-cells kill the triple Whitehead products: we can choose them to be $f^{\prime}=\left[\imath^{\prime},\left[\imath^{\prime}, \imath^{\prime \prime}\right]\right]$ and $f^{\prime \prime}=\left[\imath^{\prime \prime},\left[\imath^{\prime}, \iota^{\prime \prime}\right]\right]$. Let $B$ be the 8-skeleton $S^{3} \vee S^{3} \cup\left(e^{8} \vee e^{8}\right)$ of $M$ and $E$ be the subcomplex $S^{3} \vee S^{3} \cup_{f^{\prime}} e^{8}$. The Quillen models for the spaces $E$ and $B$ are

$$
\begin{gathered}
L_{E}=\mathbb{L}(a, b, u) ; d a=d b=0, d u=[a,[a, b]] \\
L_{B}=\mathbb{L}(a, b, u, v) ; d a=d b=0, d u=[a,[a, b]], d v=[b,[a, b]]
\end{gathered}
$$

with $|a|=|b|=2,|u|=|v|=7$.

Since $M$ is coformal, one has $\operatorname{gldim}(L)=3$ and, using [16], one sees that $\pi(B)=$ $L \amalg \mathbb{L}(w)$ with $|w|=9$, while $\pi(E)=\mathbb{L}(a, b) /([a,[a, b]])=L^{\prime}$. Therefore the attachment $f^{\prime \prime}$ to $E$ is not inert, and one easily checks it is not even lazy. However $\pi(\omega)$ factors as $L \rightarrow L \hookrightarrow L \coprod \mathbb{L}(w)$, where the map on the left is the quotient map, and thus its kernel is the ideal $K$ generated by $[b,[a, b]]$ in $L$. We claim that $K$ is a free Lie algebra, but $Q K$ is not a free $U L$-module. This can been seen through a careful analysis of the Hochschild-Serre spectral sequence of the Lie algebra extension $0 \rightarrow K \rightarrow L^{\prime} \rightarrow L \rightarrow 0$ : this spectral sequence is described by the following picture

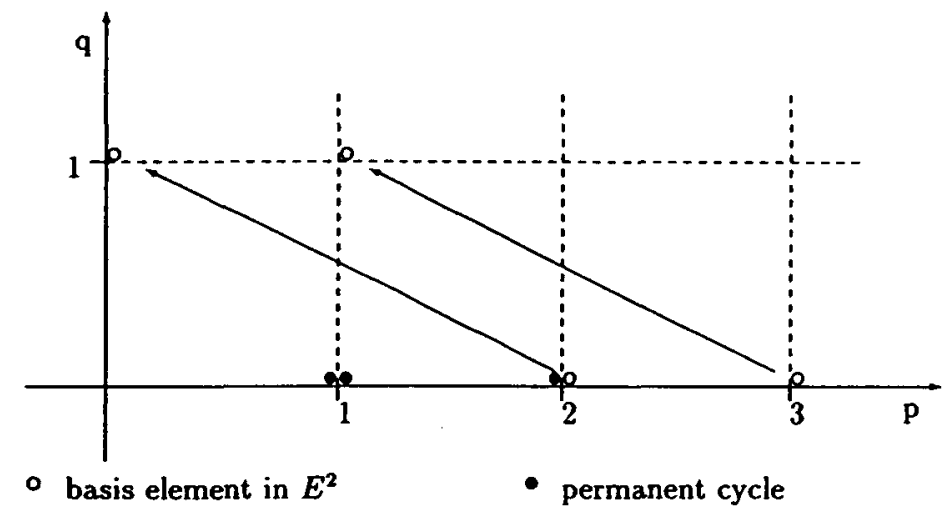

To see this, recall that the spectral sequence converges from

$$
E_{p, q}^{2}=\operatorname{Tor}_{p}^{U L}\left(\operatorname{Tor}_{q}^{U K}(\mathbb{Q}, \mathbb{Q}), \mathbb{Q}\right)
$$

to $\operatorname{Tor}_{*}^{U L^{\prime}}(\mathbb{Q}, \mathbb{Q})$. Note that $U L^{\prime}$ has two generators and one relation; the corresponding 
three elements in $\operatorname{Tor}_{1}^{U L^{\prime}}$ and $\operatorname{Tor}_{2}^{U L^{\prime}}$ form a basis for all of $\operatorname{Tor}_{*}^{U L^{\prime}}$. The latter maps injectively to $\operatorname{Tor}_{*}^{U L}(\mathbb{Q}, \mathbb{Q})$ and so these three elements are represented by permanent cycles in $E_{1,0}^{2}$ and $E_{2,0}^{2}$. It follows that in the spectral sequence $d^{2}$ maps $E_{3,0}^{2}$ onto $E_{1,1}^{2}$. Further $\operatorname{dim} E_{3,0}^{2}=\operatorname{dim} \operatorname{Tor}_{3}^{U L}(\mathbb{Q}, \mathbb{Q})=\operatorname{dim} H^{3}(\Lambda(x, y, z), d)=1$. Thus $\operatorname{dim} E_{1,1}^{2} \leqq 1$.

Consider next the $U L$-module $Q K=K /[K, K]$. It is generated by $s=[b,[a, b]]$, which satisfies the relation a.s $=0$. Since $\operatorname{dim} E_{1,1}^{2}$ is the minimal number of relations in this $U L$-module, a.s $=0$ is the unique relation and

$$
0 \rightarrow U L . a \hookrightarrow U L \rightarrow Q K \rightarrow 0
$$

is a $U L$-free resolution of $Q K$. But $E_{*, 1}^{2}=\operatorname{Tor}_{*, 1}^{U L}(\mathbb{Q}, \mathbb{Q})$ and so $E_{p, 1}^{2}=0, p \geqq 2$.

Finally, since $E_{2,0}^{\infty}=0$, we may conclude that $E_{2,0}^{2}=0$ and hence that $\operatorname{Tor}^{U K}(\mathbb{Q}, \mathbb{Q})=0$. This implies that $K$ is free.

We conclude this section with an example which shows that the holonomy action in homology does not determine the attachment, even in the inert case.

Example 4.2. Let us consider the inclusion

$$
\omega_{1}: E_{1}=S^{3} \vee S^{3} \rightarrow S^{3} \times S^{3}=B_{1}
$$

and the projection on the first factor

$$
\omega_{2}: E_{2}=\left(S^{3} \times S^{3}\right) \vee S^{5} \rightarrow S^{3} \times S^{3}=B_{2}
$$

In both cases, $B_{i}$ is obtained from $E_{i}$ by an inert attachment of a 6-cell. Let $F_{i}$ be the homotopy fibre of $\omega_{i}$; one easily checks that $F_{1}$ and $F_{2}$ both are homotopy equivalent to the same wedge of spheres, namely $\bigvee_{i>0} \bigvee_{1 \leqq j \leqq a_{1}} S_{j}^{i}$ where $S_{j}^{i}$ is a copy of the $i$-sphere and the Betti numbers $a_{i}$ have generating series $\sum_{i=1}^{\infty} a_{i} t^{i}=\left(t^{5} /\left(1-t^{2}\right)^{2}\right)$.

Since in both cases the attachment is inert, the holonomy actions are the same in homology. Actually one can even check that the two holonomy actions $\Omega B_{i} \times F_{i} \rightarrow F_{i}$ are homotopic as maps. This example shows that in general one cannot tell from the $H_{*}(\Omega B)$-structure of $H_{+}(F)$ whether the attachment kills a homology cycle. This information only shows up as a $d^{2}$-differential in the Eilenberg-Moore spectral sequence

$$
E_{p, q}^{2}=\operatorname{Tor}_{p, q}^{H_{,}(\Omega B)}\left(H_{*}(F), \kappa\right) \Rightarrow H_{p+q}(E)
$$

Indeed the differential $d_{2,4}^{2}$ is non-zero (resp. zero) for $i=1$ (resp. 2) and kills (resp. does not kill) the (module) generator in $H_{5}(F)$.

\section{Inertia and the connecting morphism}

In this section we prove Proposition 1.6. We need to consider an Adams-Hilton model of the map $\Omega B \times S^{n} \rightarrow F$ induced by the holonomy (this is why we assume $B$ to be 2-connected, although it should be possible to dispose of this restriction). Note that this 
map extends $(\delta, a): \Omega B \vee S^{n} \rightarrow F$, where $a$ lifts $\hat{f}$. We first describe the Adams-Hilton model of a product $X \times Y$, when $Y$ is an AH-suspension. Let $X$ be a 1-connected space with minimal Adams-Hilton model $A H(X)=(T(V), d)$ and $Y$ be an AH-suspension with $A H(Y)=(T(W), 0)$. We introduce the notation

$$
T(V) \otimes T(W)=T(V \oplus W \oplus s(V \otimes W))
$$

where $s$ is the suspension. For any $v \in V$ and $w \in W$, we denote $v \mid w$ the corresponding element in $s(V \otimes W)$. For any $w \in W$, we denote $. \mid w: T(V) \rightarrow T(V) \otimes T(W)$ the derivation defined by $v \mapsto v \mid w, v \in V$.

Lemma 5.1. Let $D=D_{d}$ be the derivation on $T(V) \otimes T(W)$ defined by

$$
D v=d v, D w=0, D(v \mid w)=[v, w]-d v \mid w, v \in V, w \in W .
$$

Then $D^{2}=0$, and the map

$$
\Lambda: T(V) \otimes T(W) \rightarrow T(V) \otimes T(W)
$$

defined by $v \mapsto v, w \mapsto w, v \mid w \mapsto 0$ is a surjective quasi-isomorphism. Hence $A H(X \times Y) \cong$ $(T(V) \otimes T(W), D)$.

Proof. It is immediate that $D^{2}=0$ and that $\Lambda$ is a DGA morphism. Let us consider the spectral sequence arising from the filtration of $T(V) \otimes T(W)$ by the degree in $V$. The $E^{1}$ term of this is $T(V) \otimes T(W)$ with $d^{1}(v)=0, d^{1}(w)=0, d^{1}(v \mid w)=[v, w]$, in other words $d^{1}=D_{0}$. Now $E^{1} \Lambda$ is a quasi-isomorphism by inspection, and so is $\Lambda$.

We use this model to obtain the following result, from which the "only if "part of 1.6 will easily follow:

Proposition 5.2. Let $f: X^{\circ} \rightarrow Y$ be a continuous map between 1-connected spaces. Let us assume that $Y$ is an $A H$-suspension with $\operatorname{dim} H_{+}(Y)>1$ and that the map $(f, Y): X \vee Y \rightarrow$ $Y$ extends up to homotopy to $F: X \times Y \rightarrow Y$. Then $f$ is represented by the trivial map $A H(X) \rightarrow A H(Y)$.

Proof. Let $A H(X)=(T(V), d)$ and $A H(Y)=(T(W), 0)$. The map $F$ admits a representative $\Phi:(T(V) \bigotimes T(W), D) \rightarrow(T(W), 0)$ such that $\forall w \in W, \Phi(w)=w$. Let $v \in V$ of minimal degree such that $\Phi(v) \neq 0$. Then one has for every $w \in W$

$$
0=\Phi(D(v \mid w))=[\Phi(v), w]-\Phi(d v \mid w) .
$$

But since $d v$ is decomposable, $d v \mid w$ is a sum of monomials each of which contains a term in $V_{<|v|}$, hence $\Phi(d v \mid w)=0$. Thus $\forall w \in W,[\Phi(v), w]=0$; since $\operatorname{dim} W \geqq 2$, one must have $\Phi(v)=0$, a contradiction. Therefore $\Phi=0$. 


\section{(5.3). Proof of Proposition 1.6}

Let us assume that $f$ is $p$-inert. Then by Proposition $1.2(1)$ the algebra $H_{*}(\Omega F)$ is free, i.e. $F$ is an AH-suspension. Moreover, $H_{+}(F)$ is a free $H_{*}(\Omega B)$-module of rank 1. If $H_{*}(\Omega B)=\kappa$ the map $A H(\delta)$ is obviously trivial. Otherwise $\operatorname{dim} H_{+}(F) \geqq 2$ and, since the map $(\delta, F): \Omega B \vee F \rightarrow F$ extends to the holonomy $v: \Omega B \times F \rightarrow F, \delta$ is represented by the trivial map $A H(\Omega B) \rightarrow A H(F)$ by 5.2 .

Conversely, assume that $A H(\delta): A H(\Omega B) \rightarrow A H(F)$ is homotopically trivial. Consider the composite

$$
\Omega B \times S^{n} \stackrel{\Omega B \times a}{\longrightarrow} \Omega B \times F \stackrel{\leftrightarrow}{\rightarrow} F
$$

which extends $(\Omega B, a): \Omega B \vee S^{n} \rightarrow F$. Now since $A H(\Omega B) \hookrightarrow A H(\Omega B) \bigotimes A H\left(S^{n}\right)$ is a cofibration of chain algebras, the morphism $(\kappa, A H(a)): A H(\Omega B) \bigsqcup A H\left(S^{n}\right) \rightarrow A H(F)$ extends to a morphism

$$
A H(\Omega B) \bigotimes A H\left(S^{n}\right)=T\left(s^{-1} H_{+}(\Omega B)\right) \bigotimes T\left(x_{n-1}\right) \rightarrow A H(F) .
$$

Since this map is zero on $\left(s^{-1} H_{+}(\Omega B)\right.$, it factors through the quotient of $T\left(s^{-1}(\Omega B)\right) \otimes T\left(x_{n-1}\right)$ by the ideal generated by $s^{-1} H_{+}(\Omega B)$. The quotient map has the form

$$
\bar{v}:\left(T\left(s^{-1} H_{+}\left(S^{n}\right) \oplus s^{-1}\left(H_{+}(\Omega B) \otimes H_{+}\left(S^{n}\right)\right), 0\right) \rightarrow A H(F)=\left(T\left(s^{-1} H_{+}(F)\right), d\right) .\right.
$$

But since the vector space of indecomposables $Q A H(-)$ of $A H(-)$ is naturally isomorphic to $s^{-1} H_{+}(-)$, the map $Q \bar{v}$ is an isomorphism, so is $\bar{v}$, and therefore the differential $d$ in $A H(F)$ is trivial. Thus $F$ is an AH-suspension, and $f$ is inert by $1.2(1)$.

\section{Finite depth and laziness}

Let us first recall the key observation which originally led to the definition of lazy attachments [11]: if $f$ is not lazy, the short exact sequence of $H_{*}(\Omega B)$-modules

$$
0 \rightarrow H_{+}(F) \rightarrow H_{*}(F) \rightarrow \kappa \rightarrow 0
$$

defines a non-zero element in $\operatorname{Ext}_{H_{*}(\Omega B)}^{1, n}\left(\kappa, H_{*}(\Omega B)\right)$, and therefore

$$
\operatorname{depth}\left(H_{*}(\Omega B)\right) \leqq 1 \text {. }
$$

We shall also need the notion of elliptic Hopf algebra, introduced in [7]: a Hopf algebra $H$ is said to be elliptic if $\operatorname{depth}(H)<\infty$ and $H$ has polynomial growth, i.e. there exists constants $C, k>0$ such that $\sum_{i \leqq n} \operatorname{dim} H_{i} \leqq C n^{k}$.

We can now prove Proposition 1.7.

Since $B$ has the homotopy type of a finite complex, it has finite depth by the main 
result of [6]. By a result of [10] (or [5] if $p=0$ ), the Hopf quotient $H_{*}(\Omega B) / / \operatorname{Im} H_{*}(\Omega E)$ has polynomial growth. This clearly implies that $H_{*}(\Omega B) .1 \subset H_{*}(F)$ has polynomial growth. But recall that $H_{+}(F)$ is a free $H_{*}(\Omega B)$-module of rank one. Considering its submodule $H_{*}(\Omega B) .1 \cap H_{+}(F)$ we get that $H_{*}(\Omega B)$ has a left ideal of polynomial growth. We shall now distinguish between the cases when $H_{*}(\Omega B)$ is elliptic or not; let us denote $H=H_{*}(\Omega B)$ for brevity.

Case 1: $H$ is not elliptic.

Let $\alpha$ be an element of least degree in $H_{+}$such that $\alpha \cdot 1 \neq 0$. Let $\beta$ be that unique element such that $\alpha \cdot 1=\beta \cdot a$, and let $K$ be the (primitively) generated Hopf subalgebra generated by those primitive elements in $H$ of degree $>|\alpha|$. By [8], since $H$ is not elliptic, the algebra $K$ has growth strictly greater than polynomial.

Since $|\beta|<|\alpha|$, the class of $\beta$ in $\kappa \bigotimes_{\mathrm{K}} H$ is non-zero, and since $H \cong K \otimes\left(\kappa \otimes_{\mathrm{K}} H\right)$ as $K$-modules, $K \beta$ is a free $K$-module and thus does not have polynomial growth; consequently neither does $K \alpha \cdot 1 \subset H \cdot 1 \cap H_{+}(F)$, which is impossible. Thus $\alpha \cdot 1=0$ for all $\alpha \in H_{+}$, i.e. the attachment is lazy.

Case 2: $H$ is elliptic.

If the attachment is not lazy, then $\operatorname{depth}(H) \leqq 1$ as was recalled above. Because $B$ is finite, $H$ is infinite and depth $H \neq 0$; hence depth $H=1$. But since $H$ is elliptic, Theorem $B$ in [7] shows that $H$ is isomorphic (as a vector space) to a finitely generated module over a polynomial algebra on a single generator. Therefore there exists a constant $N$ such that $\operatorname{dim} H_{k} \leqq N$ for all $k$. But by McCleary's theorem [17], this can only happen if $H^{*}(B ; \kappa)$ is monogenic, which we ruled out.

This achieves the proof of Proposition 1.7.

\section{Inert and lazy cofibrations and their fibres}

In this final section, we consider an arbitrary map $f: W \rightarrow E$ between 1-connected spaces, and its cofibre $g: E \rightarrow B=E \bigcup_{f} C W$. Let $F$ be the fibre of $g$, and let $\delta: \Omega B \rightarrow F$ be the connecting map. We may consider the following straightforward extensions of the notions of inertia and laziness:

Definition 7.1. (i) The map $f$ is p-inert if $H_{*}(\Omega g): H_{*}(\Omega E) \rightarrow H_{*}(\Omega B)$ is surjective.

(ii) The map $f$ is $p$-lazy if $H_{*}(\Omega B) \rightarrow H_{*}(F)$ is the trivial map, i.e. $H_{+}(\delta)=0$.

Note that a $p$-inert map is $p$-lazy because $\delta \circ \Omega g$ is null-homotopic. We wish to check whether our results still hold in such a general context. To begin with, it is still true-and also essentially due to Félix and Thomas-that $H_{+}(F)$ is a free $H_{*}(\Omega B)$ module under the holonomy. First note that $f: W \rightarrow E$ factors as $W \overline{\mathcal{L}}_{F} \rightarrow E$ where $\bar{f}$ is determined up to homotopy by the contraction of $f$ in $B$ through the cone on $W$. Now we have:

Proposition 7.2. The composite map 


$$
H_{*}(\Omega B) \otimes H_{+}(W) \stackrel{i d \otimes H_{*}(\bar{J})}{\longrightarrow} H_{*}(\Omega B) \otimes H_{+}(F) \stackrel{H_{*}(v)}{\longrightarrow} H_{+}\left(F^{\prime}\right)
$$

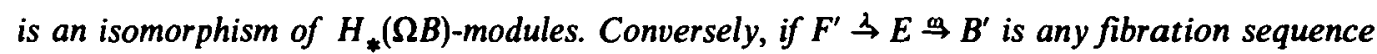
of 1-connected spaces, and if there exists a map $f^{\prime}: W \rightarrow F^{\prime}$ such that the composite

$$
H_{*}(T): H_{*}(\Omega B) \otimes H_{+}(W) \stackrel{i d \otimes H_{0}\left(S^{\prime}\right)}{\longrightarrow} H_{*}(\Omega B) \otimes H_{+}\left(F^{\prime}\right) \stackrel{H_{*}(v)}{\longrightarrow} H_{+}\left(F^{\prime}\right)
$$

is an isomorphism, then the extension $\theta: E \bigcup_{\lambda f^{\prime}} C W \rightarrow B^{\prime}$ is a homology isomorphism.

Proof. Let $F^{\prime \prime}$ be the homotopy fibre of $\theta$; by Theorem 1 in [13], there is an extension of $H_{*}(\Omega B)$-modules

$$
0 \rightarrow \operatorname{Coker} H_{*}(T) \rightarrow H_{*}\left(F^{\prime \prime}\right) \rightarrow s \operatorname{Ker} H_{*}(T) \rightarrow 0
$$

Thus when $\theta=i d_{B}, F^{\prime \prime}$ is a point, and therefore $H_{*}(T)$ is an isomorphism; conversely if the latter is an isomorphism, $H_{+}\left(F^{\prime \prime}\right)=0$, and by a standard argument $\theta: B^{\prime} \rightarrow B$ is a homology equivalence.

Let us first discuss Proposition 1.2: since Proposition 3.5 holds for any fibration sequence, the "if" part of 1.2(1) also holds in general. On the other hand, for any spaces $W$ and $B$, the inclusion $i: W \hookrightarrow W \vee B$ is clearly inert. But then $W$ is a retract of the fibre $F$ of $i$; hence $H_{*}(\Omega W)$ is a retract of $H_{*}(\Omega F)$ : therefore if $H_{*}(\Omega W)$ is not a tensor algebra, neither is $H_{*}(\Omega F)$. Thus the 'only if' part of Proposition 1.2(1) does not hold without additional hypotheses, indeed:

Proposition 7.4. The attaching map $f$ is

(1) p-inert if $F$ is an $A H$-suspension, and the converse holds if $W$ is an $A H$-suspension;

(2) p-lazy if all cup products in $H^{+}(F)$ vanish, and the converse holds if all cup products in $H^{+}(W)$ vanish.

Proof. We have just observed that the "if" statement (1) holds. Conversely, if $W$ is an AH-suspension, $A H(W)$ is the tensor algebra on $s^{-1} H_{+}(W)$ with differential zero. If $\left(y_{a}\right) \subset A H(E)$ is the image under $A H(f)$ of a basis $\left(x_{c}\right)$ of $s^{-1} H_{+}(W)$, then an Adams-Hilton model for $B$ has the form $A H(E) \bigsqcup T\left(v_{\alpha}\right)$, with $d v_{a}=y_{a}$. If $f$ is $p$-inert, it follows as in [15] that $\left(y_{\alpha}\right)$ represents an inert sequence in $H_{*}(\Omega E)$. This implies that the Hopf kernel of $H_{*}(\Omega g)$ is free. But this coincides with $H_{*}(\Omega F)$.

For (2), we observe again that the map $H_{*}(T): H_{*}(\Omega B) \otimes H_{*}(W) \rightarrow H_{*}(F)$ is a map of coalgebras. If $f$ is $p$-lazy and every element in $H_{+}(W)$ is primitive, every element in $H_{+}(F)$ can be uniquely written $\sum \alpha_{i} \alpha_{i}$ where $\left(a_{i}\right)$ is a basis of $H_{+}(W)$, and the proof of 1.2 goes on with the obvious changes.

Conversely, let us only assume that every element in $H_{+}(F)$ is primitive; if $\operatorname{dim} H_{+}(F)=1$, by 1.1(2) we are led back to the case when $W$ is a sphere, i.e. to Proposition 1.2. Thus we can assume that $H_{+}(F)$ contains two linearly independent 
elements $a$ and $b$ : choosing again an element $\beta$ of least degree in $H_{+}(\Omega B)$ such that $\beta .1 \neq 0$, computing $\Delta(\beta . a)$ and $\Delta(\beta . b)$ we get $\beta .1=\lambda a=\lambda b$, a contradiction. Hence $f$ is p-lazy.

Similarly, Proposition 1.6 readily extends to the case when $W$ is an AH-suspension:

Proposition 7.5. If $B$ is 2-connected and $W$ is a AH-suspension, $f$ is p-inert if and only if $A H(\delta)$ is homotopically trivial.

Proof. It suffices to replace $S^{n}$ by $W$ in the proof of 1.6 .

Finally, we observe that Proposition 1.7 extends to any map without additional hypotheses:

Proposition 7.6. If the fibre $F$ has finite depth, then the map $f$ is p-lazy.

Proof. Let us recall that $H_{+}(F)=\bigoplus_{\alpha} H_{*}(\Omega B) . w_{\alpha}$ is a free $H_{*}(\Omega B)$-module. If the submodule $H_{*}(\Omega B) .1 \cap H_{+}(F)$ is non-zero, its projection on one of the submodules $H_{*}(\Omega B) . w_{\alpha}$ gives a non-trivial left ideal in $H_{*}(\Omega B)$, of polynomial growth. The rest of the proof coincides with that of 1.7 .

\section{REFERENCES}

1. J. F. Adams and P. J. Hilton, On the chain algebra of a loop space, Comment Math. Helv. 30 (1955), 305-330.

2. M. Aubry and J.-M. Lemaire, Homotopies d'algèbres de Lie et de leurs algèbres enveloppantes, in Algebraic Topology \& Rational Homotopy (Springer Lect. Notes Math. 1318, 1988), 26-30.

3. H. Baues and J.-M. Lemaire., Minimal models in homotopy theory, Math. Ann. 225 (1977), 219-242.

4. A. K. Bousfield and V. K. A. M. Gugenheim, On the PL de Rham theory and rational homotopy type, Memoirs Amer. Math. Soc. 179 (1976).

5. Y. Felux, and S. Halperin, Rational LS category and its applications, Trans. Amer. Math. Soc. 273 (1982), 1-37.

6. Y. Félix, S. Halperin, J.-M. Lemaire and J.-C. Thomas, Mod $p$ loop space homology, Invent. Math. 95 (1989), 247-262.

7. Y. Felux, S. Halperin and J.-C. Thomas, Elliptic Hopf algebras, J. London Math. Soc. (2) 43 (1991), 545-555.

8. Y. Félix, S. Halperin and J.-C. Thomas, Hopf algebras of polynomial growth, J. Algebra 125 (1989), 408-417.

9. Y. Félix, S. Halperin and J.-C. Thomas, Lie algebras of polynomial growth, J. London Math. Soc. (2) 43 (1991), 556-566.

10. Y. Felix, S. Halperin and J.-C. Thomas, The Serre spectral sequence of a multiplicative fibration, preprint. 
11. Y. FÉlix and J.-M. Lemaire, On the Pontryagin algebra of the loops on a space with a cell attached, Internat. J. Math. 2 (1991), 429-438.

12. Y. Félux and J.-C. Thomas, Module d'holonomie d'une fibration, Bull. Soc. Math. France 113 (1985), 255-258.

13. Y. Félix and J.-C. Thomas, The fibre-cofibre construction and its applications, J. Pure Appl. Algebra 53 (1988), 59-69.

14. Y. Félux and J.-C. Thомas, Effet d'un attachement cellulaire dans l'homologie de l'espace des lacets, Ann. Inst. Fourier 39 (1989), 207-224.

15. S. Halperin and J.-M. Lemaire, Suites inertes dans les algèbres de Lie graduées (“Autopsie d'un meutre 2"), Math. Scand. 61 (1987), 39-67.

16. J.-M. Lemaire, Algèbres connexes et homologie des espaces de lacets (Springer Lect. Notes Math 422, 1974).

17. J. MCCleary, On the modp Betti numbers of loop spaces, Invent. Math. 87 (1987), 643-654.

18. J. W. Milnor and J. C. MoOre, On the structure of Hopf algebras, Ann. of Math. (2) 81 (1965), 211-264.

19. J. C. Moore, Differential Homological Algebra, in Proc. ICM 1970, I (Gauthier-Villars, Paris, 1971), 335-339.

20. J. C. Moore and L. Smıth, Hopf algebras and multiplicative fibrations I, Amer. J. Math. 90 (1968), 752-780.

Department of Mathematics

UNIVERSITY OF TORONTO

TORONTO

Ontario M5S lal

Canada

E-mail address: halper@lake.scar.toronto.edu
J. A. Dieudonne

URA CNRS $\mathrm{N}^{\circ} 168$

Universite de Nice Sophia-ANtipolis

F-06108 Nice Cedex 2

France

E-mail address: lemaire@math.unice.fr 